\title{
Internationale Unterschiede in den Überlebensraten
}

In den letzten 30 Jahren haben sich die Überlebensraten von

Patienten mit Bronchialkarzinom kaum verbessert, wobei teils deutliche Differenzen zwischen verschiedenen Ländern bestehen. S. Walters et al. sind nun der Frage nachgegangen, ob die unterschiedlichen Stadien zum Zeitpunkt der Diagnose dies erklären können.

Thorax 2013; 68: 551-564

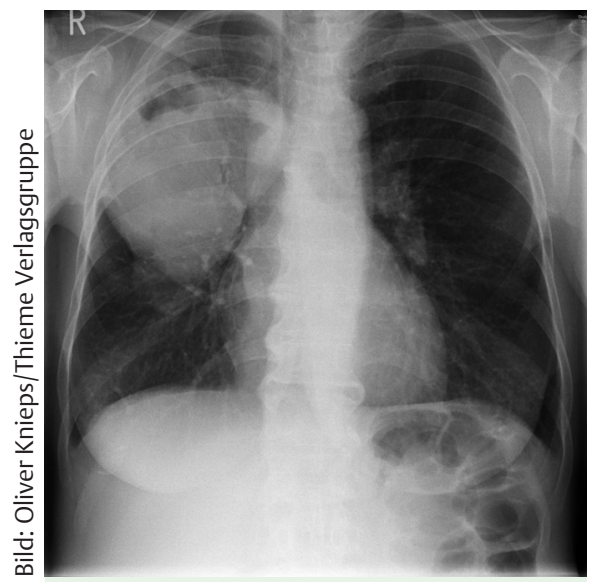

Laut Robert-Koch-Institut erkrankten 2008 allein in Deutschland 49500 Menschen an Lungenkreb. Im internationalen Vergleich schwanken die Überlebensraten.

Die Autoren sammelten hierzu Daten von Patienten aus Norwegen, Dänemark, Großbritannien, Australien, Kanada und Schweden, bei denen zwischen 2004 und 2007 ein Bronchialkarzinom diagnostiziert worden war. Sie dokumentierten unter anderem das jeweilige Tumorstadi- um bei Diagnose (TNM Klassifikation und lokale Klassifikationssysteme), wobei sie kleinzellige (SCLC) und nicht-kleinzellige Bronchialkarzinome (NSCLC) aufgrund ihrer unterschiedlichen Ätiologie und des unterschiedlichen klinischen Verhaltens getrennt analysierten. In der Folge berechneten sie die Netto-Überlebenszeiten und die Mortalität, die sie unter anderem in Relation zum Tumorstadium bei Diagnose setzten.

Insgesamt gingen 57352 Patienten in die Analyse ein. Das Durchschnittsalter lag in allen Ländern bei etwa 70 Jahren, der Anteil von Männern variierte von 52,3\% (Schweden) bis 62,2\% (Australien). Kleinzellige Bronchialkarzinome machten zwischen 12,9\% (Australien) und 18\% (Norwegen) aus, Adenokarzinome zwischen 25,2\% (Großbritannien) und 44,8\% (Schweden). Unter den NSCLC reichte der Anteil von bei Diagnose bereits metastasierten Tumoren (Stadium IV) von 46,8\% in Schweden bis 55\% in Dänemark; für SCLC lagen diese Anteile zwischen 72,1\% in Dänemark und unter 66\% in den übrigen Ländern. Die altersstandardisierten 1-Jahres-Netto-Überlebensraten von Patienten mit NSCLC variierten von $30 \%$ in Großbritannien bis zu 46\% in Schweden.

\section{Das Stadium macht den Unterschied \\ $\nabla$}

Patienten in Dänemark und Großbritannien hatten geringere Überlebensraten als in den anderen Ländern, was teilweise auf den fortgeschritteneren Tumorstadien bei Diagnosestellung beruhte. Allerdings zeigten sich auch große internationale Unterschiede im stadienspezifischen Überleben. So war das Netto-Überleben von NSCLC im Stadium I in Großbritannien um $16 \%$ geringer als in Schweden (72,5 vs. $88,4 \%$, das von NSCLC im Stadium IV war um rund $10 \%$ geringer $(15,9$ vs. $25,6 \%)$. Ähnliche Ergebnisse fanden sich auch für SCLC mit altersstandardisierten 1-Jahres-Netto-Überlebensraten zwischen 55,9\% (Großbritannien) und $71,7 \%$ (Schweden) in den Stadien I und II sowie zwischen 14,4\% (Großbritannien) und 27,6\% (Schweden) im Stadium IV.

\section{Fazit}

Auch wenn eine unterschiedliche Verteilung der Tumorstadiim bei Diagnose einen gewissen Teil der internationalen Differenzen im Überleben von Patienten mit Bronchialkarzinomen erklärt, gibt es dennoch international deutliche Unterschiede im stadienspezifischen Überleben. Dies legt nahe, dass hierbei auch noch andere Faktoren eine wichtige Rolle spielen, z.B. Unterschiede in der Behandlung, so die Autoren.

Dr. Johannes Weiß, Bad Kissingen

\section{Checklisten $\sqrt{\text { Immer auf Nummer sicher }}$}

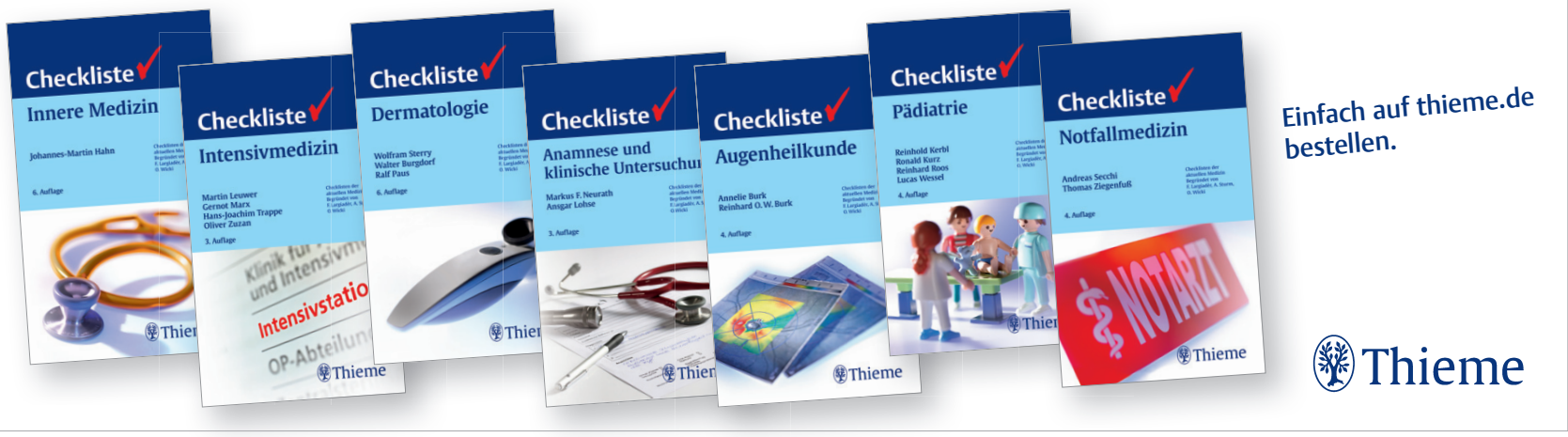

